\title{
La calidad de vida de los ancianos: revisión de literatura
}

\section{Quality of life of elderly: literature review}

\section{Qualidade de vida de idosos: revisão da literatura}

Cinara Maria Feitosa Beleza ${ }^{1}$; Maria do Livramento Fortes Figueiredo ${ }^{2}$; Claudete Ferreira de Souza Monteiro ${ }^{3}$;

Maria Eliete Batista Moura ${ }^{4}$; David Bernar Oliveira Guimarães ${ }^{5}$; Vanessa Caminha Aguiar ${ }^{6}$

${ }^{1}$ Enfermeira graduada pela Universidade Federal do Piaui/UFPI. Mestranda do Programa de Pós-Graduação Mestrado em Enfermagem da UFPI. Bolsista CAPES. Teresina, PI.

${ }^{2,3,4}$ Doutora em Enfermagem pela Universidade Federal do Rio de Janeiro. Professora da Universidade Federal do Piauí da Graduação e do Programa de Mestrado em Enfermagem. Teresina, PI.

${ }^{5}$ Acadêmico do $5^{\circ}$ período do Curso de Enfermagem da Universidade Federal do Piauí. Teresina, PI.

${ }^{6}$ Acadêmica do $6^{\circ}$ período do Curso de Enfermagem da Universidade Federal do Piauí. Teresina, PI.

Cómo citar este artículo en edición digital: Feitosa Beleza, C.Ma; Fortes Figueiredo, Ma do L; Ferreira de Souza Monteiro, C; Batista Moura, Ma E; Oliveira Guimarães. D.B; Caminha Aguiar, V. (2013) Qualidade de vida de idosos: revisão da literatura Cultura de lós Cuidados (Edición digital)17, 36. Disponible en: http://dx.doi.org/10.7184/cuid.2013.36.10.

Correspondencia: Cinara Maria Feitosa Beleza. Rua Luís Domingues 424, Centro, CEP: 65630-110, Timon (MA).

E-mail: cinara.maria@hotmail.com,tel. (86)8802-5726. Recibido: 20/05/2013. Aceptado: 3006/2013

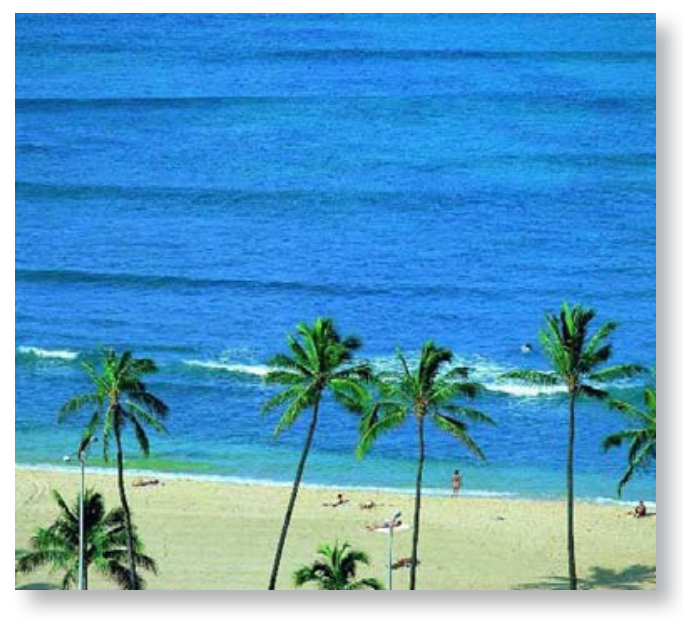

ABSTRACT

This is a literature review that investigated scientific production on quality of life of seniors. The articles were collected in SciELO database and identified the following variables: most studied topics, year and methodological approach of the publications. We identified 56 articles, of which 23 related quality of life to other health indicators, the greatest year of publication was 2011 and the quantitative approach was prevalent in the sample. The studies led to apprehend that the assessment of quality of life should be merged into nursing glimpsing the optimization of physical and mental health in the elderly.

Key words: Quality of Life, Elderly, Literature Review.

\section{RESUMEN}

Se trata de una revisión de la literatura que investigó la producción científica en la calidad de vida de los ancianos. Los artículos fueron recogidos en la base de datos SciELO y se identificaron las siguientes variables: los temas más estudiados, el año y el enfoque metodológico de las publicaciones. Se identificaron 56 artículos, 23 de los cuales la calidad de vida relacionada a los indicadores de salud, el mejor año de publicación fue 2011 y el enfoque cuantitativo era frecuente en la muestra. Los estudios llevaron a aprehender que la evaluación de la calidad de vida deberían fusionarse en enfermería vislumbrando la optimización de la salud física y mental de los ancianos.

Palabras clave: Calidad de Vida, Anciano, Revisión de la Literatura. 


\section{RESUMO}

Trata-se de uma revisão da literatura que objetivou investigar produções científicas sobre qualidade de vida de idosos. Os artigos foram levantados no banco de dados SciELO e delimitou-se as seguintes variáveis: temáticas mais estudadas, ano e abordagem metodológica das publicações. Foram identificados 56 artigos, dos quais 23 relacionavam qualidade de vida a outros indicadores de saúde, o ano de maior publicação foi 2011 e a abordagem quantitativa foi a prevalente na amostra. Os estudos propiciaram apreender que a avaliação da qualidade de vida deve ser incorporada pela enfermagem vislumbrando a otimização da saúde física e mental em idosos.

Palavras-chave: Qualidade de Vida, Idoso, Revisão da Literatura.

\section{INTRODUÇÃO}

O cenário mundial está no centro de um processo de transição demográfica única e irreversível que em conjunto com a transição epidemiológica, resulta em um dos principais acontecimentos do século 20, o envelhecimento populacional. Um fenômeno que provoca grande impacto social, ao exigir mudanças desde o nível de políticas públicas até o núcleo familiar. Dentro desse contexto torna-se, então, promissor a realização de estudos e intervenções sobre a qualidade de vida (QV) de idosos, afinal, o prolongamento da vida só pode ser considerado como uma real conquista da sociedade a medida que agrega qualidade aos anos adicionais de vida (Nasri, 2008).

Segundo a Organização Mundial de Saúde, QV é a percepção que o indivíduo tem de sua posição na vida dentro do contexto de sua cultura e do sistema de valores de onde vive, e em relação a seus objetivos, expectativas, padrões e preocupações. É um conceito muito amplo que incorpora de uma maneira complexa a saúde física de uma pessoa, seu estado psicológico, seu nível de dependência, suas relações sociais, suas crenças e sua relação com características proeminentes no ambiente (Organização Mundial de Saúde, 1994).

$\mathrm{Na}$ idade madura, essa QV excede os limites da responsabilidade pessoal e deve ser vista como um empreendimento de caráter sociocultural. Ou seja, uma velhice satisfatória não é um atributo do indivíduo biológico, psicológico ou social, mas resulta da qualidade de interação entre pessoas (Freitas, 2011).

$\mathrm{O}$ ato de envelhecer implica em mudanças constantes e saber lidar com as perdas, buscando novas aquisições durante todo o processo de envelhecimento, é o que o torna saudável. Afinal, este será cada vez mais satisfatório quanto maior for o poder do indivíduo de assimilar e não renunciar às mudanças físicas, psicológicas e sociais, adaptando-se aos novos papéis sociais que desempenhará no decorrer de sua vida (Brêtas et al, 2010).

Assim, entender como os idosos percebem o processo de envelhecimento, atrelado à noção de QV, permite aos profissionais de saúde pensar em estratégias de ações de saúde para além do enfoque na doença. Compreender o que os idosos valorizam, e como vivenciam as suas questões de saúde, relações sociais, convívio familiar, lazer, trabalho e, ainda, o acesso às suas necessidades básicas, remete ao oportuno momento de trabalhar na perspectiva da promoção da saúde e na implantação de ações que concretizem a mudança do modelo de assistência à saúde no Brasil (Paskulin et al, 2010).

Nessa perspectiva, pretendeu-se o desenvolvimento desta revisão bibliográfica, 
com o objetivo de investigar em produções científicas nacionais estudos sobre a qualidade de vida de idosos.

\section{METODOLOGIA}

Realizou-se uma revisão bibliográfica das produções científicas em saúde no Brasil, através de um levantamento na biblioteca eletrônica SciELO. Foram encontradas 120 referências ao empregar os descritores "qualidade de vida" e "idoso". Contudo, com o objetivo de ampliar a qualidade da busca e identificar o número real de publicações cujos sujeitos de estudo eram pessoas com idade de 60 anos ou mais, os resumos dos 120 artigos foram rastreados em busca das produções que discutiam exclusivamente a QV de idosos.

Foram excluídos os estudos que abordavam QV de populações não brasileiras. A exclusão desses artigos, que tratavam do tema a partir de experiências em outros países, deu-se em função da necessidade de estabelecer uma aproximação exploratória com a situação das pesquisas brasileiras.

$\mathrm{Na}$ análise empreendida, considerou-se a maneira como os autores e os sujeitos definiam QV, a utilização ou não de instrumentos específicos para determinadas patologias referidas a segmentos da população idosa, ou ainda instrumentos genéricos ligados à saúde da mesma população, contudo, sem nenhuma patologia identificada. Interessounos também observar o processo de utilização de instrumentos validados ou não para a língua portuguesa. Foram incluídos apenas os artigos originais, devido, sua maior circulação no meio acadêmico e profissional, excluindo assim, teses de doutorado, dissertações de mestrado, monografias e artigos que não continham "qualidade de vida" como descritor, o que totalizaram 56 produções.

\section{RESULTADOS}

Denota-se, no gráfico 1, que o interesse dos pesquisadores por esse assunto é recente no Brasil, ao demonstrar o crescimento das pesquisas somente a partir de 2002, com aumento significativo em 2007 (5) e 2008 (7), observando-se uma baixa no ano de 2009 (3). Entretanto, em 2010, os artigos publicados sobre o tema em questão quase que quadruplicaram (11), com destaque para 2011 que obteve o maior número de publicações (18), de caindo em 2012 (8).

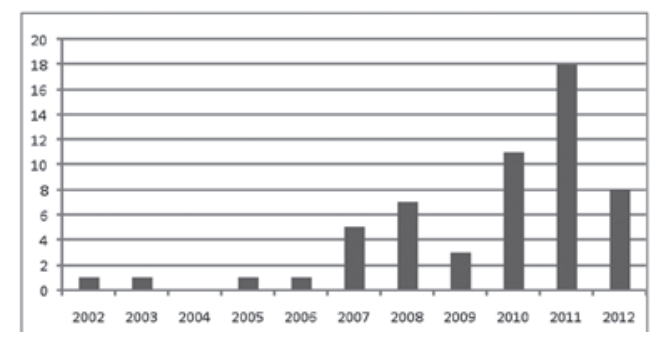

Gráfico 1. Distribuição da frequência dos artigos sobre qualidade de vida de idosos segundo o ano de publicação. Teresina/PI, 2012.

No gráfico 2 demonstra que as categorias de artigos mais frequentemente encontradas representam às pesquisas de abordagem quantitativa $(85,7 \%)$, abordagem qualitativa $(8,9 \%)$, teórico-conceitual $(3,6 \%)$ e revisão bibliográfica $(1,8 \%)$.

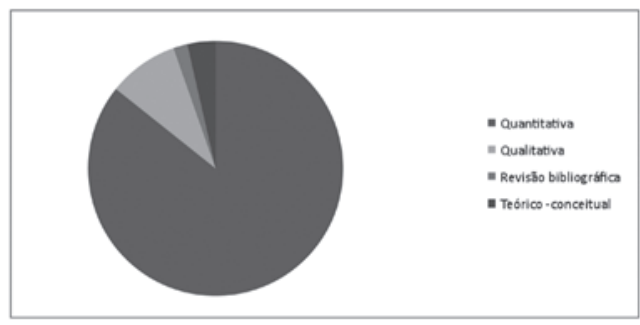

Gráfico 2. Distribuição dos artigos sobre qualidade de vida de idosos segundo abordagem metodológica. Teresina/PI, 2012 . 
Quanto às temáticas mais encontradas, a Tabela 1 demonstra o expressivo interesse dos pesquisadores para a relação entre variáveis independentes diversas e QV $(41,1 \%)$ e a avaliação da própria QV (35,4\%). Destacouse ainda, as avaliações de intervenções para melhorar a QV (12,5\%), os significados de QV (10,7\%) e, em menor percentual, (7,1\%) a elaboração e análise de instrumentos de coleta de dados para a avaliação da QV em idosos. e bem-estar (Lima; Portela, 2010. Paskulin et al, 2010). Além de ser, constantemente, usada como um importante parâmetro na busca dos fatores relacionados à maior expectativa de vida e os determinantes da boa QV - para estimular -, e os de má qualidade - para intervir (Maués, 2010).

Assim também, os pesquisadores têm dado ênfase a essa temática motivados pela busca de estabelecer as razões e os padrões do envelhecimento disfuncional, buscando a descrição sistemática das condições que antecederiam e contribuiriam para mudanças provocadas pelo envelhecimento e para a determinação da QV de idosos (Beckert; Irigaray; Trentini, 2012).

Tabela 1. Distribuição dos artigos sobre qualidade de vida segundo as temáticas das publicações. Teresina/PI, 2012.

\section{DISCUSSÃO}

Ao analisar as publicações quanto ao ano, os dados mostraram (Fig. 1) o recente e crescente aumento do número de publicações em estudo sobre a temática QV de idosos. Ao realizar um corte temporal entre 2002 a 2008, observa-se que a quantidade de artigos sobre o assunto foi setuplicada em 2008, tendo em vista o ano de 2002. E, em destaque têm-se os últimos três anos (2010, 2011 e 2012) que, em suma, compreendem $66,1 \%$ dos artigos publicados.

A medição de QV na velhice tem sido grandemente valorizada nas últimas décadas, em função da necessidade de se conhecer os resultados e a eficiência da aplicação de intervenções de saúde, análise de políticas públicas e avaliação de cuidados prestados, principalmente no domínio da funcionalidade

\begin{tabular}{lcr} 
Para avaliar & os estudos \\
relativos às & \multicolumn{2}{c}{ abordagens } \\
metodológicas, & a $r$ & Figura
\end{tabular}

2 demonstra maior índice de pesquisas quantitativas. Este tipo de estudo utiliza a coleta e análise de dados para responder às questões de pesquisa e testar hipóteses estabelecidas previamente, e confia na medição numérica, na contagem e frequentemente no uso de estatística para estabelecer com exatidão os padrões de comportamento de uma população. A abordagem quantitativa associa-se aos experimentos, as pesquisas com questões fechadas ou a estudos em que se empregam instrumentos de medição padronizados (Sampieri; Collado; Lucio, 2006).

Identificou-se também o interesse pela pesquisa qualitativa, a qual se aplica o estudo da história, das relações, das representações, das crenças, das percepções e das opiniões, produtos das interpretações que os humanos 


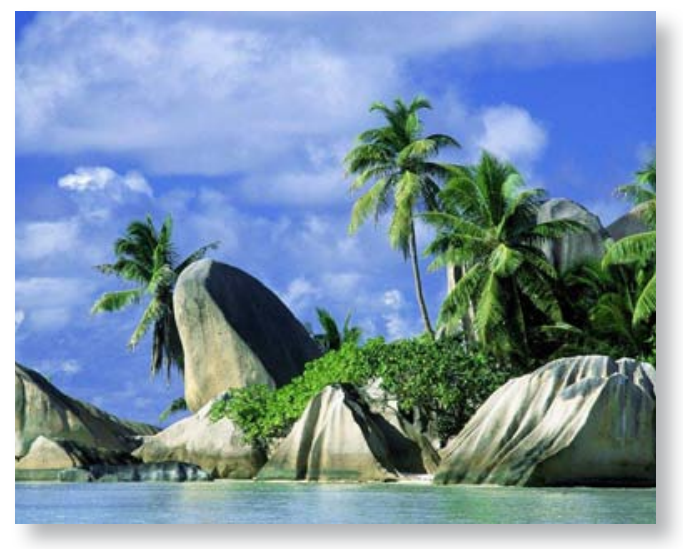

fazem a respeito de como vivem, constroem seus artefatos e a si mesmos. As abordagens qualitativas conformam-se melhor nas investigações de grupos e segmentos delimitados e focalizados, de histórias sociais sob a ótica dos atores, de relações e para análises de discursos e de documentos (Minayo, 2008).

A abordagem metodológica de estudos teórico-conceituais tem como característica abrangente, discussões conceituais a partir da literatura, revisões bibliográficas. Assim como, modelagens conceituais, baseadas na percepção e experiências do autor (Berto; Nakano, 1999).

Por fim, a revisão de literatura apresenta como proposta o processo de levantamento e análise do que já foi publicado sobre um tema ou um problema de pesquisa escolhidos. A revisão contribui para obter informações sobre a situação atual do tema pesquisado; para conhecer as publicações existentes sobre este e os aspectos que já foram abordados e para verificar as opiniões similares e diferentes relacionadas ao mesmo (Silva; Menezes, 2005).

Corroborando com este estudo, uma pesquisa desenvolvida em 2007, com corte temporal 2000-2005 e temática sobre a produção científica na área de saúde sobre QV no Brasil, observou que grande parte dos estudos encontrados pretendeu avaliar a QV de grandes grupos ou populações e, instrumentos quantitativos de avaliação de QV foram utilizados, por isso, a abordagem quantitativa foi a mais utilizada pelos autores, seguida da abordagem qualitativa (Oliveira, 2007).

Com base no estudo de Lopes e Rabelo (2006), que apresentou um panorama geral de artigos nacionais e internacionais sobre QV e velhice, foi considerado o agrupamento dos artigos em cinco grupos e suas respectivas análises.

Na Tabela 1, observou-se que a maioria dos artigos versava sobre pesquisas que investigavam a relação entre $\mathrm{QV}$ e variáveis independentes diversas, as quais estavam relacionadas à saúde física, mental e psicológica e a aspectos sociais e ambientais. Essa temática reflete a preocupação em avaliar quais fatores influenciam de maneira positiva ou negativa os vários domínios de vida dos idosos. Dos 23 artigos componentes desse grupo, alguns investigaram a influência de variáveis relacionadas à saúde física sobre a QV desse grupo etário, como, por exemplo, capacidade funcional, tratamento hemodialítico, doença crônica, fratura vertebral, estado nutricional, insuficiência cardíaca, tontura, osteoartrite de joelho, déficit visual. Outros artigos analisaram as relações entre QV e variáveis relacionadas à saúde mental e psicológica, tais como, depressão, sintomas neuropsiquiátricos, sintomas psicossomáticos, sono e religiosidade. Por fim, os demais estudos examinaram a influência de variáveis relacionadas a aspectos sociais e ambientais como a institucionalização, tipos de comportamentos, nível de instrução, status socioeconômico e fatores sociossanitários.

Em segundo lugar, aparecem as pesquisas que objetivaram avaliar a QV de populações específicas, dentre as quais foram descritas 
as de idosos octagenários, idosos com insuficiência cardíaca, idosos com doença de Alzheimer, idosos na comunidade, idosos participantes de grupos de lazer e idosos com doença pulmonar obstrutiva crônica. Nessa categoria, a QV foi analisada mediante avaliação subjetiva de domínios específicos previamente estabelecidos. As conclusões dos trabalhos classificados nessa temática indicam que o intuito de tais avaliações é o de conhecer os caminhos a serem percorridos e os pontos em que as intervenções devem ser feitas para possibilitar uma QV ótima aos idosos.

As pesquisas presentes na terceira categoria investigaram os significados relacionados à $\mathrm{QV}$ segundo os participantes dos estudos. Isto é, foi analisado o que o idoso define como QV e o que considerava importante para tê-la, e não uma avaliação sobre a satisfação com determinados domínios de vida preestabelecidos. Os artigos presentes nesse grupo demonstraram o interesse em analisar os elementos que os idosos julgam significativos para ter ou manter uma QV satisfatória.

Em quarto lugar, ficaram os estudos relacionados à metodologia de pesquisa, incluindo nessa categoria investigações que objetivavam o desenvolvimento e a validação de instrumentos, a avaliação de instrumentos já existentes e a comparação entre as medidas das percepções de idosos e cuidadores sobre a QV dos primeiros. O número de artigos englobados nessa categoria indica a ocorrência do processo de construção do saber sobre o assunto e o reconhecimento da importância de medidas fidedignas e consistentes nesse processo.

Por fim, a última categoria engloba as pesquisas sobre a avaliação de intervenções para melhorar a QV. Os artigos da temática 5 reforçam a ideia de que existe uma preocupação dos profissionais envolvidos com os idosos relacionada à prática de como melhorar a $\mathrm{QV}$, ao revolver as investigações em torno de intervenções, seja através de programas de apoio social, seja através de intervenções cirúrgicas.

\section{CONCLUSÃO}

Foi possível assinalar pontos importantes a serem considerados como a necessidade de direcionar a atenção para a manutenção da QV da população idosa. De modo geral, os resultados dessa investigação corroboram o reconhecimento de que o construto QV demarca um avanço significativo na inclusão do processo saúde-doença na perspectiva ampliada e situacional do sujeito.

Os levantamentos realizados não tiveram a intenção de efetuar uma pesquisa bibliográfica aprofundada sobre o tema QV, e sim apresentar uma visão geral sobre a produção científica nacional. E, dentre esta, o enfoque prevalente foi a da relação entre QV e variáveis independentes diversas.

Em referência ao período compreendido entre 2002-2012, foram encontrados 120 artigos que abordavam o referido tema, publicados, em sua maioria, nos anos de 2010, 2011 e 2012.

Ao categorizar os artigos, evidenciou-se a predominância da pesquisa de abordagem quantitativa seguida da abordagem qualitativa. Uma minoria referiu-se à pesquisa teóricoconceitual e revisão da literatura.

A tarefa em avaliar a QV de idosos vem sendo realizada, visto o crescimento de artigos desenvolvidos nos últimos três anos. Logo, espera-se que a divulgação dos resultados desse estudo seja veículo de motivação para os profissionais de saúde a trabalharem essa temática na sua prática cotidiana, vislumbrando a otimização da saúde mental e da aptidão física em idosos, bem como 
à intervenção voltada para os benefícios individuais e sociais que podem sobrevir da melhoria da QV.

\section{REFERÊNCIAS}

- Beckert M, Irigaray TQ, Trentini CM. (2012) Qualidade de vida, cognição e desempenho nas funções executivas de idosos. Est. psicol. (Campinas), 29(2), 155-162. Disponível em: http://www.scielo. br/scielo.php?script $=$ sci_arttext $\&$ pid $=$ S0103-166X2 012000200001\&lng=en\&tlng=pt. 10.1590/S0103166X2012000200001.

- Berto RMVS, Nakano DN. (1999) A produção científica nos anais do encontro nacional de engenharia de produção: um levantamento de métodos e tipos de pesquisa. Prod. 1999, 9(2): 65-76. Disponível em: http://www.scielo.br/scielo.php?script=sci_ arttext\&pid=S0103-65131999000200005\&lng=en\&tlng =pt. 10.1590/S0103-65131999000200005.

- Brêtas ACP et al. (2010) Quem mandou ficar velho e morar na rua? Rev. esc. enferm. USP, 44(2): 476-81. Disponível em: $\quad$ http://www.scielo.br/scielo.php?script=sci_ arttext\&pid=S0080-62342010000200033\&lng=en\&tlng $=$ pt. $10.1590 /$ S0080-62342010000200033.

- Freitas VFFS. (2011) Qualidade de vida do idoso no município de Herval. Rev. enferm. saúde, 1(1): 156-63. Disponível em: http://www.ufpel.edu.br/revistas/index. php/enfermagemesaude/article/viewFile/54/39.

- Lima MJB, Portela MC. (2010) Elaboração e avaliação da confiabilidade de um instrumento para medição da qualidade de vida relacionada à saúde de idosos independentes. Cad. Saúde Pública, 26(8): 1651-62. Disponível em: http://www.scielo. br/scielo.php?script=sci_arttext $\&$ pid $=$ S0102-311X2 010000800018\&lng=en\&tlng=pt. $\quad 10.1590 /$ S0102311 X2010000800018.

- Lopes ESL, Rabelo DF. (2006) Qualidade de vida na velhice: indicadores nacionais e internacionais. Rev. Kairós, 9(2): 209-26. Disponível em: http://bases.bireme. br/cgi-bin/wxislind.exe/iah/online/?IsisScript=iah/iah. $\mathrm{xis} \& \mathrm{~b}$ ase $=$ LILACS\&nextAction $=\operatorname{lnk} \&$ lang $=\mathrm{p} \&$ indexS earch $=$ ID\&exprSearch $=469437$ \&label $=$ Qualidade $\% 20$ de\%20vida\%20na $\% 20$ velhice:\%20indicadores $\% 20$ nacionais $\% 20 \mathrm{e} \% 20$ internacionais.

- Maués CR. (2010) Avaliação da qualidade de vida: comparação entre idosos jovens e muito idosos. Rev. Soc. Bras. Clín. Med, 8(5): 405-10. Disponível em: http://bases.bireme.br/cgi-bin/wxislind.exe/iah/ online/?IsisScript=iah/iah.xis\&src=google\&base $=$ LILA CS\&lang $=$ p\&nextAction $=\operatorname{lnk} \& \operatorname{exprSearch}=561602 \&$ in dexSearch=ID.

- Minayo MCS. (2008) O desafio do conhecimento: pesquisa qualitativa em saúde. $11^{\text {a }}$ ed. São Paulo: Hucitu. - Nasri F. (2008) O envelhecimento populacional no Brasil. Einstein, 6(1): 4-6. Disponível em: http://bases.bireme. br/cgi-bin/wxislind.exe/iah/online/?IsisScript=iah/iah. xis\&src $=$ google \&base $=$ LILACS\&lang $=p \&$ nextAction $=1$ nk\&exprSearch=516986\&indexSearch=ID.

- Oliveira CJ. (2007) Análise da produção científica na área da saúde sobre qualidade de vida no Brasil entre 2000 e 2005: um estudo bibliográfico. Rev. eletrônica enferm, 9(2): 496-505. Disponível em: http://www.fen. ufg.br/fen_revista/v9/n2/v9n2a16.htm.

- Organização Mundial de Saúde. (1994) Declaração Elaborada pelo Grupo de Trabalho da Qualidade de Vida da OMS: glossário de Promoção de Saúde da OMS de 1998 in: OMS/HPR/HEP/ 98.i Genebra: Organização Mundial da Saúde, 1994.

- Paskulin LMG et al. (2010) Percepção de pessoas idosas sobre qualidade de vida. Acta paul. Enferm, 23(1): 101-07. Disponível em: http://www.scielo. br/scielo.php?script=sci_arttext $\&$ pid=S010321002010000100016\&lng=en\&tlng=pt. 10.1590/S010321002010000100016.

- Sampieri RH, Collado CF, Lucio PB. (2006) Metodologia de Pesquisa. $3^{\text {a }}$ ed. São Paulo: McGraw-Hill.

- Silva EL, Menezes EM. (2005) Metodologia da pesquisa e elaboração de dissertação. $4^{\mathrm{a}}$ ed. rev. atual. Florianópolis: UFSC. 